\title{
Prognostic and clinical impact of PIK3CA mutation in gastric cancer: pyrosequencing technology and literature review
}

\author{
Kazuto Harada', Yoshifumi Baba', Hironobu Shigaki ${ }^{1}$, Takatsugu Ishimoto ${ }^{1}$, Keisuke Miyake ${ }^{1}$, Keisuke Kosumi ${ }^{1}$, \\ Ryuma Tokunaga', Daisuke Izumi ${ }^{1}$, Mayuko Ohuchi ${ }^{1}$, Kenichi Nakamura', Yuki Kiyozumi', Junji Kurashige ', \\ Masaaki Iwatsuki ${ }^{1}$, Yuji Miyamoto', Yasuo Sakamoto ${ }^{1}$, Naoya Yoshida', Masayuki Watanabe ${ }^{2}$ and Hideo Baba ${ }^{1 *}$
}

\begin{abstract}
Background: Phosphatidylinositol-4,5-bisphosphate 3-kinase, catalytic subunit alpha (PIK3CA) mutations that activate the PI3K/AKT signaling pathway have been observed in several types of carcinoma and have been associated with patient prognosis. However, the significance of PIK3CA mutations in gastric cancer remains unclear. This retrospective study investigated the relationship between PIK3CA mutations and clinical outcomes in patients with gastric cancer. Additionally, we reviewed the rate of PIK3CA mutations in gastric cancer and the association between PIK3CA mutations and prognosis in human cancers.

Methods: The study included 208 patients with gastric cancer who underwent surgical resection at Kumamoto University Hospital, Japan, between January 2001 and August 2010. Mutations in PIK3CA exons 9 and 20 were quantified by pyrosequencing assays.

Results: PIK3CA mutations were detected in 25 (12 \%) of the 208 patients. Ten patients had c.1634A > G (p.E545G), 10 had c.1624G > A (p.E542K), 13 had c.1633G > A (p.E545K), nine had c.3139C > T (p.H1047R), and 1 had c.3140A > G (p. H1047Y) mutations. PIK3CA mutations were not significantly associated with any clinical, epidemiologic, or pathologic characteristic. Kaplan-Meier analysis showed no significant differences in disease-free survival (log rank $P=0.84)$ and overall survival (log rank $P=0.74$ ) between patients with and without PIK3CA mutations.
\end{abstract}

Conclusions: Mutations in PIK3CA did not correlate with prognosis in patients with gastric cancer, providing additional evidence for the lack of relationship between the two.

Keywords: PIK3CA mutation, Gastric cancer, Pyrosequencing, Prognosis

\section{Background}

Gastric cancer is the third leading cause of cancer deaths in the world, with 723,000 patients dying of gastric cancer in 2012 [1]. Elucidating the biological pathways leading to the development of gastric carcinoma is crucial because accumulation of genetic alterations has been shown to result in tumor development [2]. Genetic alterations involving proteins along several signaling pathways, such as the constitutive activation of receptor tyrosine kinases and G-protein-coupled receptors, and

\footnotetext{
* Correspondence: hdobaba@kumamoto-u.ac.jp

${ }^{1}$ Department of Gastroenterological Surgery, Graduate School of Medical Science, Kumamoto University, 1-1-1 Honjo, Kumamoto 860-8556, Japan
} Full list of author information is available at the end of the article
GTP-binding proteins to adaptor proteins, could lead to activation of the phosphoinositide 3-kinase (PI3K)-AKT pathway [3-6]. As PI3K signaling plays essential roles in cell growth, metabolism, survival, metastasis, and resistance to chemotherapy, the PI3K-AKT pathway has been considered extremely important in the carcinogenic process [7-10].

Mutations in the phosphatidylinositol-4,5-bisphosphate 3-kinase, catalytic subunit alpha (PIK3CA) gene, which encodes the p110 catalytic subunit of PI3K, have been found in several types of carcinoma $[11,12]$. The hot spots of PIK3CA mutations have been located at five sites in exons 9 and 20 [13]. These mutations activate the PI3K/AKT signaling 
pathway, activating downstream signaling pathways, and thereby contribute to carcinogenesis [13]. PIK3CA mutations in several types of human cancer have been associated with patient prognosis. However the impact of PIK3CA mutations on prognosis varies among tumor types [14].

The significance of PIK3CA mutations in gastric cancer remains unclear. Although several reports have

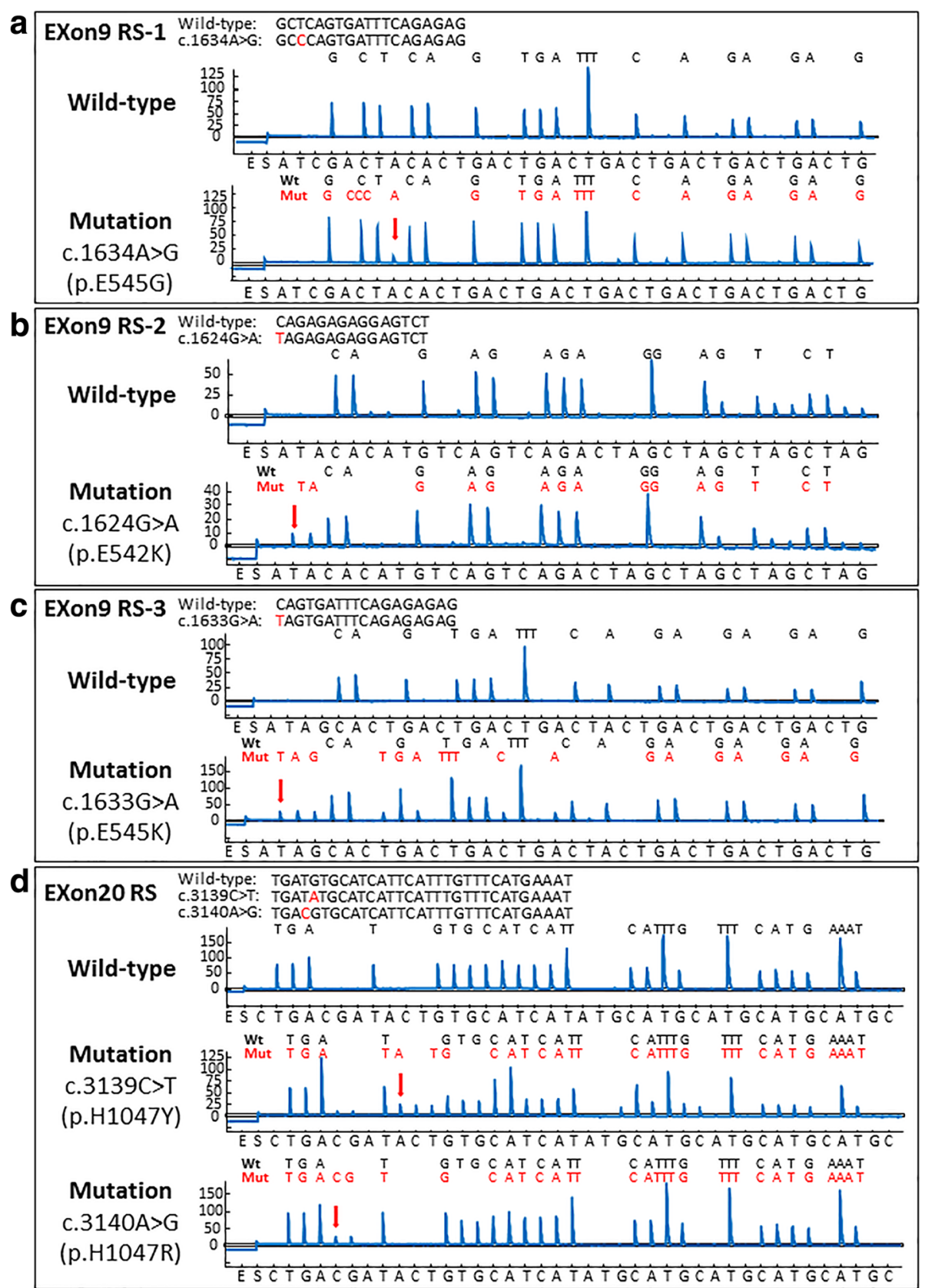

Fig. 1 PIK3CA exon 9 and exon 20 pyrograms (antisense strand). a Wild-type exon 9 sequenced with the 9-RS1 primer and the $C .1634 A>G$ mutation (arrow), causing a shift in the reading frame and a new peak at A (arrowhead), which serves as quality assurance. b Wild-type exon 9 sequenced with the 9-RS2 primer and the C.1624G > A mutation (arrow), causing a shift in the reading frame and a new peak at A (arrowhead). c Wild-type exon 9 sequenced with the 9-RS3 primer and the c.1633G > A mutation (arrow), causing a shift in the reading frame and new peaks (arrowheads). d Wild-type exon 20 sequenced with the exon 20 primer and the c.3139C > T mutation (arrow), causing a shift in the reading frame and a new peak at T (arrowhead). Wild-type exon 20 sequenced with the exon 20 primer and the c.3140A > G mutation (arrow), causing a shift in the reading frame and a new peak at G (arrowhead). Mut, mutant; WT, wild-type 
shown a relationship between PIK3CA mutations and prognosis, relative few patients were analyzed and findings differ among studies. This study used pyrosequencing to evaluate PIK3CA mutations in patients with gastric cancer at our hospital, as well as determining the relationship between PIK3CA mutations and patient prognosis. Additionally, we reviewed the rate of PIK3CA mutations in gastric cancer and the association between PIK3CA mutations and prognosis in human cancers.

\section{Methods}

\section{Study subjects}

This study retrospectively enrolled 208 gastric cancer patients who underwent resection at Kumamoto University Hospital between January 2001 and August 2010. Patients who underwent palliative resection and/or whose tissue samples were unavailable were excluded, but patients positive on peritoneal washing cytology were included. Patients were followed-up at 1- to 3-

Table 1 PIK3CA mutational status and clinical features in gastric cancers

\begin{tabular}{|c|c|c|c|c|}
\hline \multirow[t]{3}{*}{ Features } & \multirow{3}{*}{$\begin{array}{l}\text { Total } \\
n=208\end{array}$} & \multicolumn{2}{|c|}{ PIK3CA mutation } & \multirow[t]{3}{*}{$p$ value } \\
\hline & & Mutant & Wild-Type & \\
\hline & & $n=25(12 \%)$ & $n=183(88 \%)$ & \\
\hline Age (year) & & & & 0.30 \\
\hline Mean \pm SE & 208 & $67.4 \pm 2.1$ & $69.8 \pm 0.8$ & \\
\hline Sex & & & & 0.41 \\
\hline Male & 148 & $16(11)$ & $132(89)$ & \\
\hline Female & 60 & $9(15)$ & $51(85)$ & \\
\hline Location & & & & 0.22 \\
\hline Upper & 75 & $13(17)$ & $62(83)$ & \\
\hline Middle & 65 & $6(9)$ & $59(91)$ & \\
\hline Lower & 68 & $6(9)$ & $62(91)$ & \\
\hline Tumor depth & & & & 0.09 \\
\hline $\mathrm{T} 1$ & 105 & $18(17)$ & $87(83)$ & \\
\hline $\mathrm{T} 2$ & 29 & $2(6)$ & $29(94)$ & \\
\hline T3 & 42 & $2(5)$ & $42(95)$ & \\
\hline $\mathrm{T} 4$ & 25 & $3(11)$ & $25(89)$ & \\
\hline Lymph node involvement & & & & 0.73 \\
\hline Absent & 135 & $17(13)$ & $118(87)$ & \\
\hline Present & 73 & $8(11)$ & $65(83)$ & \\
\hline Stage & & & & 0.78 \\
\hline । & 123 & $17(14)$ & $106(86)$ & \\
\hline$\|$ & 40 & $4(10)$ & $36(90)$ & \\
\hline III & 26 & $2(8)$ & $24(92)$ & \\
\hline IV & 19 & $2(11)$ & $17(89)$ & \\
\hline Lauren classification & & & & 0.79 \\
\hline Intestinal & 138 & $16(12)$ & $122(88)$ & \\
\hline Diffuse & 70 & $9(13)$ & $61(87)$ & \\
\hline Venous invasion & & & & 0.05 \\
\hline Absent & 111 & $18(16)$ & $93(84)$ & \\
\hline Present & 95 & $7(7)$ & $88(93)$ & \\
\hline Lymphatic invasion & & & & 0.40 \\
\hline Absent & 117 & $16(14)$ & $101(86)$ & \\
\hline Present & 91 & $9(10)$ & $82(90)$ & \\
\hline LINE-1 methylation level (\%) & & & & 0.19 \\
\hline Mean \pm SE & 208 & $74.9 \pm 2.6$ & $71.2 \pm 1.0$ & \\
\hline
\end{tabular}


month intervals until death or 31 March 2015, whichever came first. Disease-free survival was defined as the time from the date of surgery to the date of cancer recurrence or death. Tumors were staged according to the American Joint Committee on Cancer Staging Manual (7th edition) [15]. Overall survival was defined as the time from the date of the operation to the date of death. All procedures followed were in accordance with the ethical standards of the responsible committee on human experimentation (institutional and national) and with the Helsinki Declaration of 1964 and later versions. Informed consent or substitute for it was obtained from all patients for being included in the study. The study procedures were approved by the Ethics Committee for Epidemiological and General Research at the Faculty of life Science, Kumamoto University (Approval number: Ethic 559). Throughout this article, the definition of "prognostic marker" is consistent with REMARK Guidelines [16].

\section{Genomic DNA extraction}

Genomic DNA was extracted from paraffin-embedded tissue specimens of surgically resected gastric cancers. Tumors areas were marked on hematoxylin and eosin stained slides by one pathologist (Y. Baba). Genomic DNA was extracted from tumor lesions enriched with neoplastic cells, without adjacent normal tissue, using FFPE Kits (Qiagen, Duesseldorf, Germany).

\section{Pyrosequencing for PIK3CA mutations}

PIK3CA mutations were evaluated as previously described [14]. The exon 9 PCR primers were $5^{\prime}$-biotin-AACAGCTC AAAGCAATTTCTACACG-3' (forward, PIK3CA 9-F) and 5' -ACCTGTGACTCCATAGAAAATCTTT-3' (reverse,
PIK3CA9-R). The exon 20 PCR primers were 5'-biotinCAAGAGGCTTTGGAGTATTTCA-3' (forward, PIK3CA 20-F) and 5'-CAATCCATTTTTGTTGTCCA-3' (reverse, PIK3CA 20-R). In the PIK3CA exon 9 pyrosequencing assays, the presence of mutations was routinely confirmed using three different sequencing primers and by the creation of a frameshifted reading of a mutant sequence relative to a wild-type sequence. The primers PIK3CA 9-RS1 (5'-CCATAGAAAATCTTTCTCCT-3'; nucleotide dispensation order, ATCGACTACACTGACTGACTGACTGACTGACTGACTG), PIK3CA 9-RS2 (5' TTCTCCTTGCTTCAGTGATTT-3'; nucleotide dispensation order, ATACACATGTCAGTCAGACTAGCTAGCTAGCTAG) and PIK3CA 9-RS3 (5' TAGAAAATCTTTCTCCTGCT-3'; nucleotide dispensation order, ATAGCACTGACTGACTGACTACTGACTGACTGACTG) could detectc.1634A > G, c.1624 $\mathrm{A}>\mathrm{G}$, and c.1624G $>\mathrm{A}$ mutations, respectively. For PIK3CA exon 20, the primer PIK3CA 20-RS (5'GTTGTCCAGCCACCA-3'; nucleotide dispensation order, CTGACGATACTGTGCATCATATGCATGCAT GCATGCATGC) was used to detect the mutations c.3140A $>$ G and c.3139C $>$ T. Nucleotide dispensation orders were designed so that if any of the common mutations were present, it caused a shift in the reading frame and resulted in an additional peak or peaks following the mutated nucleotide. Representative pyrograms of wild-type and mutant exons 9 and 20 are shown in Fig. 1.

\section{Statistical methods}

The results were statistically analyzed by JMP software (Version 9, SAS Institute, Cary, NC, USA). All p-values were two-sided. The means were compared by $t$ test,

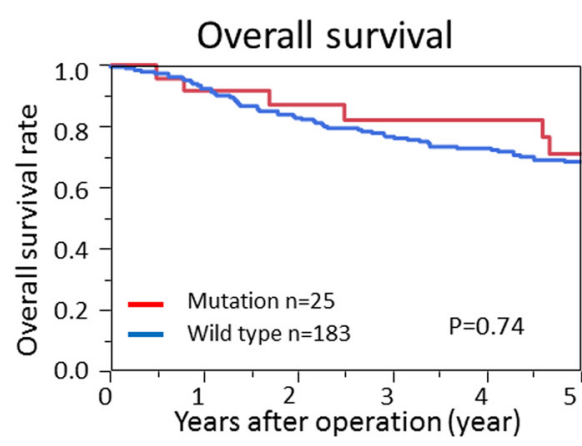

Number at risk

\begin{tabular}{|c|c|c|c|c|c|c|}
\hline Years & 0 & 1 & 2 & 3 & 4 & 5 \\
\hline mutation & 25 & 23 & 20 & 18 & 17 & 16 \\
\hline Wild type & 183 & 164 & 147 & 129 & 101 & 69 \\
\hline
\end{tabular}

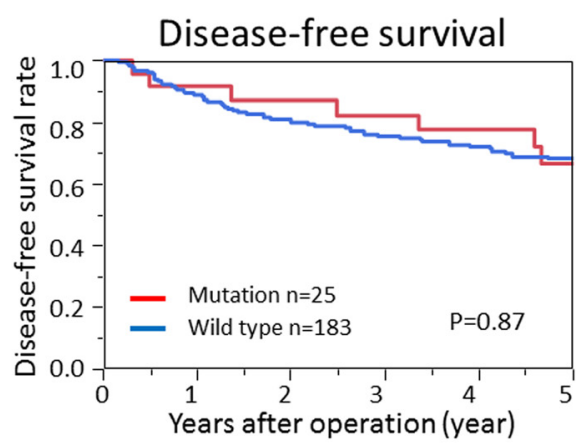

Number at risk

\begin{tabular}{|c|c|c|c|c|c|c|}
\hline Years & 0 & 1 & 2 & 3 & 4 & 5 \\
\hline mutation & 25 & 23 & 19 & 18 & 16 & 12 \\
\hline Wild type & 183 & 156 & 137 & 122 & 97 & 66 \\
\hline
\end{tabular}

Fig. 2 Kaplan-Meier curves showing the relationship of PIK3CA mutational status with overall survival (left) and disease-free survival (right) in gastric cancer patients 


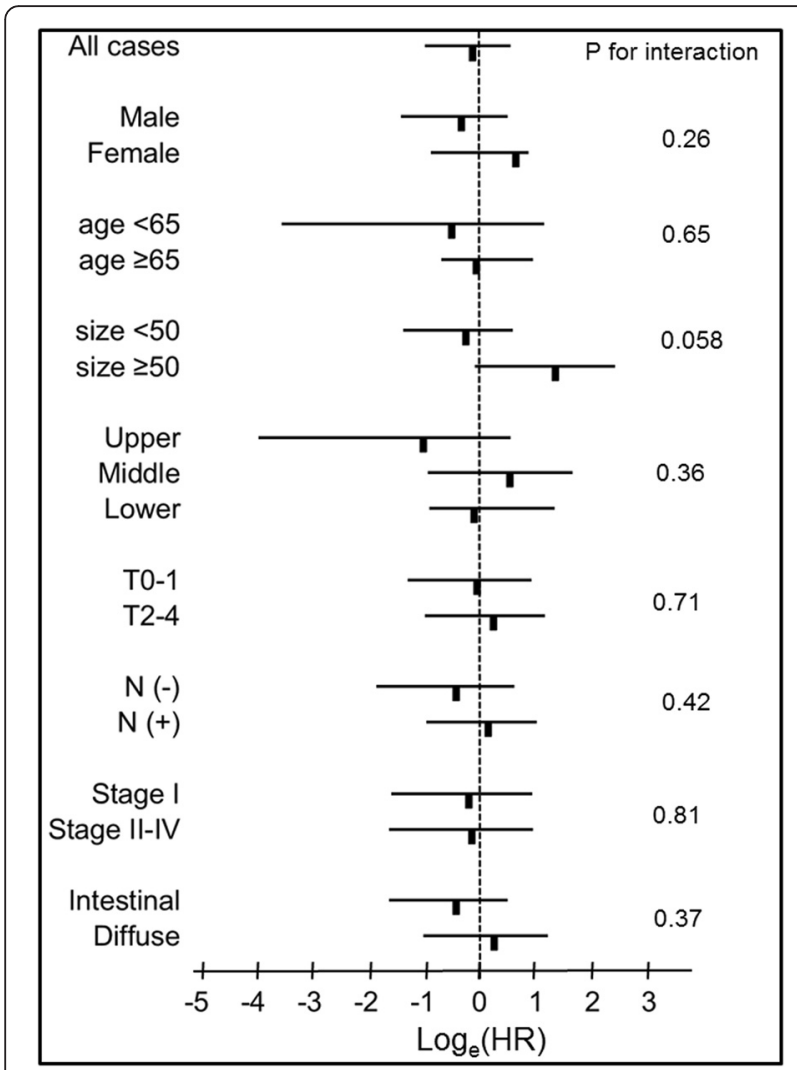

Fig. 3 PIK3CA mutations and overall survival in various strata. The $\log _{\mathrm{e}}$ (adjusted HRs) plot of overall survival in the PIK3CA mutation vs. PIK3CA wild-type group. The $95 \% \mathrm{Cl}$ is also shown

assuming unequal variances. The proportional differences among the characteristics were evaluated by Pearson's chi-squared test $\left(\chi^{2}\right)$. Survival time was determined by the Kaplan-Meier method and compared using the log-rank test. The hazard ratio for PIK3CA mutations on mortality was assessed by Cox regression modeling. To assess whether any of these variables was associated with the relationship between the PIK3CA mutations and prognosis, they were cross-multiplied with PIK3CA mutations and subjected to the Wald test.

\section{Results}

PIK3CA mutational status in gastric cancer

Of the 208 patients who had undergone curative resection of gastric cancer, 25 (12\%) were found pyrosequencing technology to have PIK3CA exon 9 and 20 mutations (Fig. 1). Ten patients were positive for c.1634A > G (p.E545G), 10 for c.1624G > A (p.E542K), 13 for c. c.1633G > A (p.E545K), nine for c. 3139 C $>$ T (p.H1047R), and one for c. 3140A > G (p.H1047Y) mutations. Three tumors were positive for mutations at all three nucleotides in exon 9, three were positive for mutations at two nucleotides in exon 9. Fifteen samples were positive for mutations in exon 9 alone, four for mutations in exon 20 alone, and six were positive for mutations in both exons.

\section{PIK3CA mutations and patient characteristics}

PIK3CA mutations were not significantly associated with any clinical, epidemiologic, or pathologic characteristic (Table 1). We previously reported that the long interspersed nucleotide element-1 (LINE-1) methylation level in gastric cancer was a surrogate marker of global DNA methylation and genome instability $[17,18]$. However, LINE-1 methylation level was not associated with PIK3CA mutations (Table 1).

\section{PIK3CA mutations and patient survival}

We assessed the influence of PIK3CA mutations on clinical outcome in patients with curatively resected

Table 2 Previous studies of PIK3CA mutations in gastric cancer

\begin{tabular}{|c|c|c|c|c|}
\hline Sample number & Mutation rate & Exons evaluated & Method & References \\
\hline 163 & $5.5 \%$ & 9 and 20 & Direct sequencing & [36] \\
\hline 263 & $16 \%$ & 9 and 20 & Direct sequencing & [27] \\
\hline 12 & $25 \%$ & 18 and 20 & Sanger method & [6] \\
\hline 47 & $11 \%$ & 9 and 20 & ABI prism 377 automated sequencer & [37] \\
\hline 94 & $4.3 \%$ & 9 and 20 & Direct sequencing & [38] \\
\hline 185 & $6.5 \%$ & 9,18 and 20 & PCR - SSCP & [39] \\
\hline \multirow[t]{2}{*}{34} & $6.0 \%$ & 9 and 20 & High-Resolution & [40] \\
\hline & & & Melt Analysis & \\
\hline 295 & $24 \%$ & All exon & Mut Sig CV & [41] \\
\hline \multirow[t]{2}{*}{140} & $4.0 \%$ & 9 and 20 & ABI PRISM 3100 & [42] \\
\hline & & & Genetic Analyzer & \\
\hline 231 & $8.7 \%$ & 1,9 and 20 & Pyrosequencing & [26] \\
\hline 104 & $7.7 \%$ & 9 and 20 & Direct sequencing & [28] \\
\hline
\end{tabular}


gastric cancer. During follow-up of the 208 patients, 32 patients experienced gastric cancer recurrence and 69 died. The median follow-up time for censored patients was 5.0 years. Kaplan-Meier analysis showed no significant differences in disease-free survival (log rank $P=0.84)$ and overall survival ( $\log \operatorname{rank} P=0.74$ ) between patients with PIK3CA mutations and those with PIK3CA wild type (Fig. 2).

\section{Interaction between PIK3CA mutations and other variables in survival analyses}

We also examined whether the influence of PIK3CA mutations on overall survival was modified by any clinical or pathological variable. All tested variables did not significantly interact with the relationship between PIK3CA mutations and overall survival ( $\mathrm{p}$ for all interactions $>0.05$, Fig. 3). Moreover, analysis of various strata showed no significant difference in hazard ratio for overall survival between patients with PIK3CA mutations and those with PIK3CA wild type (Fig. 3).

\section{Discussion}

PIK3CA mutations and subsequent activation of the PI3K/AKT pathway play an essential role in cancer cell signaling pathways, involving growth factors, cytokines, and other cellular stimuli associated with human

Table 3 Studies on prognostic significance of PIK3CA mutations in several types of cancers

\begin{tabular}{|c|c|c|c|c|c|}
\hline Cancer type & Sample number & Mutation rate (\%) & Mutation effect & Prognosis & References \\
\hline \multirow[t]{6}{*}{ Colon cancer } & 1212 & 16 & Unfavorable & Adjusted CS HR & {$[31]$} \\
\hline & & & & $3.51(1.28-9.62)$ & \\
\hline & 743 & 14.5 & Unfavorable & Adjusted OS HR & {$[25]$} \\
\hline & & & & $3.30(1.46-7.45)$ & \\
\hline & 450 & 18 & Unfavorable & Adjusted CS HR & [29] \\
\hline & & & & $2.03(1.15-3.57)$ & \\
\hline \multirow[t]{2}{*}{ Rectal cancer } & 240 & 7.9 & Unfavorable & Adjusted RS HR & {$[32]$} \\
\hline & & & & $3.4(1.2-9.2)$ & \\
\hline \multirow[t]{2}{*}{ Endometrial cancer } & 1063 & 16.2 & Unfavorable & Not-adjusted RS HR & [33] \\
\hline & & & & $2.18(1.09-4.39)$ & \\
\hline \multirow[t]{6}{*}{ Breast cancer } & 687 & 25.3 & favorable & Not-adjusted CS HR & {$[34]$} \\
\hline & & & & $0.88(0.58-1.34)$ & \\
\hline & 439 & 32.5 & Not significant & Adjusted CS HR & {$[30]$} \\
\hline & & & & $0.7(0.4-1.2)$ & \\
\hline & 188 & 28.7 & favorable & Adjusted RS HR & {$[35]$} \\
\hline & & & & $0.42(0.20-0.92)$ & \\
\hline \multirow[t]{4}{*}{ Esophageal cancer } & 406 & 7.4 & Not significant & Adjusted OS HR & {$[43]$} \\
\hline & & & & $1.072(0.79-1.44)$ & \\
\hline & 219 & 21 & favorable & Adjusted OS HR & [14] \\
\hline & & & & $0.35(0.10-0.90)$ & \\
\hline \multirow[t]{4}{*}{ Lung cancer } & 1117 & 3.4 & Not significant & OS log-lank & {$[44]$} \\
\hline & & & & $P=0.442$ & \\
\hline & 235 & 3.4 & Not significant & OS log-lank & {$[45]$} \\
\hline & & & & $P=0.15$ & \\
\hline \multirow[t]{8}{*}{ Gastric cancer } & 263 & 15.9 & Not significant & Adjusted OS HR & [27] \\
\hline & & & & $1.1(0.7-1.7)$ & \\
\hline & 231 & 8.7 & Not significant & Not-adjusted OS HR & {$[26]$} \\
\hline & & & & $1.37(0.68-3.26)$ & \\
\hline & 104 & 7.7 & Not significant & OS log-lank & {$[28]$} \\
\hline & & & & $P=0.96$ & \\
\hline & 208 & 12 & Not significant & Not-adjusted OS HR & This study \\
\hline & & & & $0.73(0.58-2.57)$ & \\
\hline
\end{tabular}


neoplasms $[11,12,19,20]$. This study examined the prognostic impact of PIK3CA mutations on survival in patients with gastric cancer. Mutations in PIK3CA exons 9 and 20 were observed in 25 of the 208 (12\%) gastric cancer patients. However, overall survival and diseasefree survival were similar in patients with and without PIK3CA mutations, indicating that PIK3CA mutations do not affect mortality after resection of gastric cancer.

PIK3CA exons 9 and 20 mutation accounted for over the $80 \%$ of the mutations [6]. Exon 9 mutation exist in helical domain, and exon 20 mutation exist in kinase domain, leading to the activating of AKT pathway and then stimulating the ability of invasion and migration [13]. Especially, E545K mutation disrupts inhibitory interaction between p110 and SH2 domain in p85 [21]. Importantly, Baba et al. have shown the relationship between tumor phosphorylated AKT expression and PIK3CA exons 9 and 20 mutation in 717 colorectal cancer [22]. The oncogenic role of mutations other than hot spot mutation has not been fully investigated. Interestingly, some tumor samples had multiple mutations on exon 9 and 20. Some group have also detected several different PIK3CA mutations in same sample by using next-generation sequencing and Sanger Sequencing. It is possible that one sample harvest three or two different mutations in the same gene $[23,24]$. In addition, PIK3CA mutation can coexist with KRAS, EGFR and BRAF [25]. Thus, PIK3CA mutation has been involved in molecular pathway of cancer.

Other studies have reported PIK3CA mutations in 4-25\% of gastric cancers (Table 2). The largest cohort was assessed by the TOGA study group, finding that the PIK3CA mutation rate was $24 \%$, or about twice as high as in the current study. However, that study evaluated mutations in all exons. A study in a Japanese population found that the mutation rate in PIK3CA exons 1,9 , and 20, as determined by pyrosequencing, was $8.7 \%$ [26]. Directed sequencing found that the mutation rate in PIK3CA exons 9 and 20 was $7.7-15.9 \%[27,28]$. Thus, the PIK3CA mutation rate observed in the current study (12 \%) was comparable to rates previously reported. Interestingly, the PIK3CA mutation rate in gastric cancer is less than that in colon and breast cancers $[29,30]$.

Previous studies evaluating the association between PIK3CA mutations and prognosis in human cancers have yielded variable results (Table 3). PIK3CA mutations have been associated with a better prognosis in patients with breast and esophageal cancers, but with a poorer prognosis in patients with colon, rectum, and endometrial cancers [31-35]. These differences may be due to differences in tumor histology. Discovering the mechanisms of this discrepancy is imperative for future projects.
The current study found that PIK3CA mutations were not associated with survival in patients with gastric cancer, in agreement with three previous studies [26-28]. Because of their relatively large sample sizes, including this study that included samples from over 200 samples, these findings, taken together, confirm the lack of relationship between PIK3CA mutations and prognosis in gastric cancer patients. These findings, however, require confirmation by large independent cohort studies.

\section{Conclusions}

In conclusion, mutations in PIK3CA exons 9 and 20 were observed in 25 of the 208 (12\%) gastric cancer patients by pyrosequencing assays. We found that PIK3CA mutations did not correlate with prognosis in patients with gastric cancer, providing additional evidence for the lack of relationship between the two.

\section{Abbreviations}

LINE-1, long interspersed nucleotide element-1; PI3K, phosphoinositide 3-kinase; PIK3CA, phosphatidylinositol-4,5-bisphosphate 3-kinase, catalytic subunit alpha

\section{Acknowledgements}

None.

\section{Funding}

None.

Availability of data and materials

All datasets on which the conclusions of the paper are either presented in the main manuscript.

\section{Authors' contributions}

Conception and design: $\mathrm{KH}, \mathrm{YB}, \mathrm{SS}, \mathrm{MW}$ and $\mathrm{HB}$; acquisition of data: $\mathrm{KH}, \mathrm{YB}$, TI, KK, RT, DI, MO, KN, YKi, JK, MI, SI, YM, YS and NY; analysis and interpretation of data: $\mathrm{KH}$ and $\mathrm{YB}$; manuscript writing: $\mathrm{KH}, \mathrm{YB}$, and $\mathrm{HB}$. All authors approved the final manuscript.

\section{Competing interests}

The authors declare that they have no competing interests.

Consent for publication

Not Applicable.

\section{Ethics approval and consent to participate}

All procedures followed were in accordance with the ethical standards of the responsible committee on human experimentation (institutional and national) and with the Helsinki Declaration of 1964 and later versions. The study procedures were approved by the Ethics Committee for

Epidemiological and General Research at the Faculty of life Science, Kumamoto University (Approval number: Ethic 559). Informed consent for this study was obtained from all patients.

\section{Author details}

${ }^{1}$ Department of Gastroenterological Surgery, Graduate School of Medical Science, Kumamoto University, 1-1-1 Honjo, Kumamoto 860-8556, Japan. ${ }^{2}$ Department of Gastroenterological Surgery, Cancer Institute Hospital, Japanese Foundation for Cancer Research, Tokyo, Japan.

Received: 19 December 2015 Accepted: 15 June 2016 Published online: 07 July 2016 


\section{References}

1. Ferlay J, Soerjomataram I, Dikshit R, Eser S, Mathers C, Rebelo M, et al. Cancer incidence and mortality worldwide: sources, methods and major patterns in GLOBOCAN 2012. Int J Cancer. 2015;136(5):359-86.

2. Deng N, Goh LK, Wang H, Das K, Tao J, Tan IB, et al. A comprehensive survey of genomic alterations in gastric cancer reveals systematic patterns of molecular exclusivity and co-occurrence among distinct therapeutic targets. Gut. 2012;61(5):673-84

3. Handschick K, Beuerlein K, Jurida L, Bartkuhn M, Muller H, Soelch J, et al. Cyclin-dependent kinase 6 is a chromatin-bound cofactor for NF-kappaBdependent gene expression. Mol Cell. 2014;53(2):193-208.

4. Cantley LC. The phosphoinositide 3-kinase pathway. Science. 2002; 296(5573):1655-7.

5. Katso R, Okkenhaug K, Ahmadi K, White S, Timms J, Waterfield MD. Cellular function of phosphoinositide 3-kinases: implications for development, homeostasis, and cancer. Annu Rev Cell Dev Biol. 2001;17:615-75.

6. Samuels Y, Wang Z, Bardelli A, Silliman N, Ptak J, Szabo S, et al. High frequency of mutations of the PIK3CA gene in human cancers. Science. 2004;304(5670):554.

7. Willems L, Tamburini J, Chapuis N, Lacombe C, Mayeux P, Bouscary D. PI3K and mTOR signaling pathways in cancer: new data on targeted therapies. Curr Oncol Rep. 2012;14(2):129-38.

8. Sun M, Wang G, Paciga JE, Feldman Rl, Yuan ZQ, Ma XL, et al. AKT1/ PKBalpha kinase is frequently elevated in human cancers and its constitutive activation is required for oncogenic transformation in $\mathrm{NIH} 3 \mathrm{~T} 3$ cells. Am J Pathol. 2001;159(2):431-7.

9. Itoh N, Semba S, Ito M, Takeda H, Kawata S, Yamakawa M. Phosphorylation of Akt/PKB is required for suppression of cancer cell apoptosis and tumor progression in human colorectal carcinoma. Cancer. 2002;94(12):3127-34.

10. Matsuoka T, Yashiro M. The Role of PI3K/Akt/mTOR Signaling in Gastric Carcinoma. Cancers. 2014;6(3):1441-63.

11. Engelman JA, Luo J, Cantley LC. The evolution of phosphatidylinositol 3-kinases as regulators of growth and metabolism. Nat Rev Genet. 2006;7(8):606-19.

12. Manning BD, Cantley LC. AKT/PKB signaling: navigating downstream. Cell. 2007;129(7):1261-74.

13. Samuels Y, Diaz Jr LA, Schmidt-Kittler O, Cummins JM, Delong L, Cheong et al. Mutant PIK3CA promotes cell growth and invasion of human cancer cells. Cancer Cell. 2005:7(6):561-73.

14. Shigaki H, Baba Y, Watanabe M, Murata A, Ishimoto T, Iwatsuki M, et al. PIK3CA mutation is associated with a favorable prognosis among patients with curatively resected esophageal squamous cell carcinoma. Clin Cancer Res. 2013;19(9):2451-9.

15. Washington K. 7th edition of the AJCC cancer staging manual: stomach. Ann Surg Oncol. 2010;17(12):3077-9.

16. McShane LM, Altman DG, Sauerbrei W, Taube SE, Gion M, Clark GM Reporting recommendations for tumor marker prognostic studies (REMARK) J Natl Cancer Inst. 2005:97(16):1180-4

17. Shigaki H, Baba Y, Watanabe M, Murata A, Iwagami S, Miyake K, et al. LINE-1 hypomethylation in gastric cancer, detected by bisulfite pyrosequencing, is associated with poor prognosis. Gastric Cancer. 2013;16(4):480-7.

18. Cordaux R, Batzer MA. The impact of retrotransposons on human genome evolution. Nat Rev Genet. 2009;10(10):691-703.

19. Gymnopoulos M, Elsliger MA, Vogt PK. Rare cancer-specific mutations in PIK3CA show gain of function. Proc Natl Acad Sci U S A. 2007:104(13):5569-74.

20. Ikenoue T, Kanai F, Hikiba Y, Obata T, Tanaka Y, Imamura J, et al. Functional analysis of PIK3CA gene mutations in human colorectal cancer. Cancer Res. 2005;65(11):4562-7.

21. Miled N, Yan Y, Hon WC, Perisic O, Zvelebil M, Inbar $Y$, et al. Mechanism of two classes of cancer mutations in the phosphoinositide 3-kinase catalytic subunit. Science. 2007;317(5835):239-42.

22. Baba Y, Nosho K, Shima K, Hayashi M, Meyerhardt JA, Chan AT, et al. Phosphorylated AKT expression is associated with PIK3CA mutation, low stage, and favorable outcome in 717 colorectal cancers. Cancer. 2011;117(7): 1399-408.

23. Dirican E, Kaya Z, Gullu G, Peker I, Ozmen T, Gulluoglu BM, et al. Detection of PIK3CA gene mutations with HRM analysis and association with IGFBP-5 expression levels in breast cancer. Asian Pac J Cancer Prev. 2014;15(21): 9327-33.

24. Arsenic R, Treue D, Lehmann A, Hummel M, Dietel M, Denkert C, Budczies J. Comparison of targeted next-generation sequencing and Sanger sequencing for the detection of PIK3CA mutations in breast cancer. BMC Clin Pathol. 2015:15:20.

25. De Roock W, Claes B, Bernasconi D, De Schutter J, Biesmans B, Fountzilas G, et al. Effects of KRAS, BRAF, NRAS, and PIK3CA mutations on the efficacy of cetuximab plus chemotherapy in chemotherapy-refractory metastatic colorectal cancer: a retrospective consortium analysis. Lancet Oncol. 2010; $11(8): 753-62$

26. Sukawa $Y$, Yamamoto $H$, Nosho K, Kunimoto H, Suzuki H, Adachi Y, et al. Alterations in the human epidermal growth factor receptor 2 phosphatidylinositol 3-kinase-v-Akt pathway in gastric cancer. World J Gastroenterol. 2012;18(45):6577-86.

27. Barbi S, Cataldo I, De Manzoni G, Bersani S, Lamba S, Mattuzzi S, et al. The analysis of PIK3CA mutations in gastric carcinoma and metanalysis of literature suggest that exon-selectivity is a signature of cancer type. J Exp Clin Cancer Res. 2010:29:32.

28. Lee H, Hwang IS, Choi IJ, Kang YN, Park KU, Lee JH. Are PIK3CA Mutation and Amplification Associated with Clinicopathological Characteristics of Gastric Cancer? Asian Pac J Cancer Prev. 2015;16(11):4493-6.

29. Ogino S, Nosho K, Kirkner GJ, Shima K, Irahara N, Kure S, et al. PIK3CA mutation is associated with poor prognosis among patients with curatively resected colon cancer. J Clin Oncol. 2009;27(9):1477-84.

30. Kalinsky K, Jacks LM, Heguy A, Patil S, Drobnjak M, Bhanot UK, et al. PIK3CA mutation associates with improved outcome in breast cancer. Clin Cancer Res. 2009;15(16):5049-59.

31. Liao X, Morikawa T, Lochhead P, Imamura Y, Kuchiba A, Yamauchi M, et al. Prognostic role of PIK3CA mutation in colorectal cancer: cohort study and literature review. Clin Cancer Res. 2012;18(8):2257-68.

32. He Y, Van't Veer LJ, Mikolajewska-Hanclich I, van Velthuysen ML, Zeestraten EC, Nagtegaal ID, et al. PIK3CA mutations predict local recurrences in rectal cancer patients. Clin Cancer Res. 2009:15(22):6956-62.

33. Garcia-Dios DA, Lambrechts D, Coenegrachts L, Vandenput I, Capoen A, Webb PM, et al. High-throughput interrogation of PIK3CA, PTEN, KRAS, FBXW7 and TP53 mutations in primary endometrial carcinoma. Gynecol Oncol. 2013:128(2):327-34

34. Loi S, Michiels S, Lambrechts D, Fumagalli D, Claes B, Kellokumpu-Lehtinen $\mathrm{PL}$, et al. Somatic mutation profiling and associations with prognosis and trastuzumab benefit in early breast cancer. J Natl Cancer Inst. 2013;105(13): 960-7.

35. Maruyama N, Miyoshi Y, Taguchi T, Tamaki Y, Monden M, Noguchi S. Clinicopathologic analysis of breast cancers with PIK3CA mutations in Japanese women. Clin Cancer Res. 2007;13(2):408-14

36. Takahashi N, Yamada Y, Taniguchi H, Fukahori M, Sasaki Y, Shoji H, et al. Clinicopathological features and prognostic roles of KRAS, BRAF, PIK3CA and NRAS mutations in advanced gastric cancer. BMC Res Notes. 2014;7:271.

37. Velho S, Oliveira C, Ferreira A, Ferreira AC, Suriano G, Schwartz Jr S, et al. The prevalence of PIK3CA mutations in gastric and colon cancer. Eur J Cancer. 2005:41(11):1649-54.

38. Li VS, Wong CW, Chan TL, Chan AS, Zhao W, Chu KM, et al. Mutations of PIK3CA in gastric adenocarcinoma. BMC Cancer. 2005:5:29.

39. Lee JW, Soung YH, Kim SY, Lee HW, Park WS, Nam SW, et al. PIK3CA gene is frequently mutated in breast carcinomas and hepatocellular carcinomas. Oncogene. 2005;24(8):1477-80

40. Moehler M, Mueller A, Trarbach T, Lordick F, Seufferlein T, Kubicka S, et al. Cetuximab with irinotecan, folinic acid and 5-fluorouracil as first-line treatment in advanced gastroesophageal cancer: a prospective multi-center biomarker-oriented phase II study. Ann Oncol. 2011;22(6):1358-66.

41. Cancer Genome Atlas Research N. Comprehensive molecula characterization of gastric adenocarcinoma. Nature. 2014;513(7517):202-9.

42. Chong ML, Loh M, Thakkar B, Pang B, lacopetta B, Soong R. Phosphatidylinositol-3-kinase pathway aberrations in gastric and colorectal cancer: meta-analysis, co-occurrence and ethnic variation. Int J Cancer. 2014; 134(5):1232-8

43. Wang $L$, Shan $L$, Zhang $S$, Ying J, Xue L, Yuan $Y$, et al. PIK3CA gene mutations and overexpression: implications for prognostic biomarker and therapeutic target in Chinese esophageal squamous cell carcinoma. PLoS ONE. 2014:9(7):e103021.

44. Wang L, Hu H, Pan Y, Wang R, Li Y, Shen L, et al. PIK3CA mutations frequently coexist with EGFR/KRAS mutations in non-small cell lung cancer and suggest poor prognosis in EGFR/KRAS wildtype subgroup. PLOS ONE. 2014;9(2):e88291. 